\title{
Algebraically Closed Fields
}

Thierry Coquand

September 2010 


\section{Algebraic closure}

In the previous lecture, we have seen how to "force" the existence of prime ideals, even in a weark framework where we don't have choice axiom

Instead of "forcing" the existence of a point of a space (a mathematical object), we are going to "force" the existence of a model (a mathematical structure) 


\section{Forcing/Beth models/Kripke models}

1955 Beth models

1962 Cohen forcing

1964 Kripke models

1964 sheaf models, site models, topos

1966 Boolean valued models 


\section{Algebraic closure}

The first step to build a closure of a field $k$ is to show that we can build a splitting field of any polynomial $P$ in $k[X]$. We have to build an extension of $k$ where $P$ decomposes in linear factor.

As we have seen in the first lecture, there is no hope to do this effectively, even for the polynomial $X^{2}+1$ 


\section{Algebraically closed fields}

Language of ring. Theory of ring, equational

Field axioms $1 \neq 0$ and

$x=0 \vee \exists y .1=x y$

Algebraically closed $\exists x \cdot x^{n}+a_{1} x^{n-1}+\cdots+a_{n}=0$

For an extension of $k$ we add the diagram of $k$

$a \neq b$ stands for $\neg(a=b)$ and $\neg \varphi$ stands for $\varphi \rightarrow \perp$ 


\section{Algebraically closed fields}

We show effectively the consistency of this theory by defining a forcing relation $R \Vdash \varphi$

where $R$ is a finitely presented $k$-algebra

Thus $R$ is of the form $k\left[X_{1}, \ldots, X_{n}\right] /\left\langle P_{1}, \ldots, P_{m}\right\rangle$

This forcing relation will be sound: $\vdash \varphi$ implies $R \Vdash \varphi$

We shall have $R \Vdash 1=0$ iff $1=0$ in $R$ 


\section{Algebraically closed fields}

$R$ represents a state of knowledge about the (ideal) model: we have a finite number of indeterminates $X_{1}, \ldots, X_{n}$ and a finite number of conditions $P_{1}=\cdots=P_{m}=0$ 


\section{Site model}

\section{Elementary covering}

fields $R \rightarrow R\left[a^{-1}\right]$ and $R \rightarrow R /\langle a\rangle$ : we force $a$ to be invertible or to be 0

algebraically closed fields: we add $R \rightarrow R[X] /\langle p\rangle$ where $p$ is a monic non constant polynomial

An arbitrary covering is obtained by iterating elementary coverings (in all these cases, we obtain only finite coverings) 


\section{Site model}

One defines a forcing relation $R \Vdash \varphi$ by induction on $\varphi$

$R \Vdash \varphi\left(a_{1}, \ldots, a_{n}\right) \rightarrow \psi\left(a_{1}, \ldots, a_{n}\right)$ iff $S \Vdash \varphi\left(f\left(a_{1}\right), \ldots, f\left(a_{n}\right)\right)$ implies $S \Vdash \psi\left(f\left(a_{1}\right), \ldots, f\left(a_{n}\right)\right)$ for any map $f: R \rightarrow S$

$R \Vdash \forall x . \varphi\left(a_{1}, \ldots, a_{n}, x\right)$ iff for any map $R \rightarrow S$ and any element $b$ in $S$ we have $S \Vdash \varphi\left(f\left(a_{1}\right), \ldots, f\left(a_{n}\right), b\right)$

$R \Vdash \varphi_{0} \wedge \varphi_{1}$ iff we have $R \Vdash \varphi_{0}$ and $R \Vdash \varphi_{1}$ 


\section{Site model}

$R \Vdash \exists x . \varphi\left(a_{1}, \ldots, a_{n}, x\right)$ iff we have a covering $f_{i}: R \rightarrow R_{i}$ and elements $b_{i}$ in $R_{i}$ such that $R_{i} \Vdash \varphi\left(f_{i}\left(a_{1}\right), \ldots, f_{i}\left(a_{n}\right), b_{i}\right)$

$R \Vdash \varphi_{0}\left(a_{1}, \ldots, a_{n}\right) \vee \varphi_{1}\left(a_{1}, \ldots, a_{n}\right)$ iff we have a covering $f_{i}: R \rightarrow R_{i}$ and $R_{i} \Vdash \varphi_{0}\left(f_{i}\left(a_{1}\right), \ldots, f_{i}\left(a_{n}\right)\right)$ or $R_{i} \Vdash \varphi_{1}\left(f_{i}\left(a_{1}\right), \ldots, f_{i}\left(a_{n}\right)\right)$ for all $i$ 


\section{Site model}

$R \Vdash t\left(a_{1}, \ldots, a_{n}\right)=u\left(a_{1}, \ldots, a_{n}\right)$ iff we have a covering $f_{i}: R \rightarrow R_{i}$ and $t\left(f_{i}\left(a_{1}\right), \ldots, f_{i}\left(a_{n}\right)\right)=u\left(f_{i}\left(a_{1}\right), \ldots, f_{i}\left(a_{n}\right)\right)$ in each $R_{i}$

$R \Vdash \perp$ iff we have a covering $f_{i}: R \rightarrow R_{i}$ and $1=0$ in each $R_{i}$ 


\section{Site model}

In this way, we "force"

$R \Vdash a=0 \vee \operatorname{inv}(a)$ theory of fields, where $\operatorname{inv}(a)$ is $\exists x . a x=1$

$R \Vdash \exists x \cdot x^{n}+a_{1} x^{n-1}+\cdots+a_{n}=0$ theory of algebraically closed fields 


\section{Finitely presented $k$-algebra}

Any map $R \rightarrow S$ between two finitely presented $k$-algebra can be seen as a composition of two basic operations

-adding a new indeterminate $R \rightarrow R[X]$

-adding a new relation $R \rightarrow R /\langle p\rangle$ 


\section{Exploding nodes}

If an element $a$ has already an inverse in $R$ then $R /\langle a\rangle$ is trivial

Similarly if $a$ is nilpotent in $R$ then $R\left[a^{-1}\right]$ is trivial

If $R$ is trivial, i.e. $1=0$ in $R$, then we have $R \Vdash \perp$ and $R \Vdash \varphi$ for all $\varphi$ 


\section{Soundness Theorem}

Theorem: If we have $\varphi_{1}, \ldots, \varphi_{n} \vdash \varphi$ in intuitionistic natural deduction and if $R \Vdash \varphi_{1}, \ldots, R \Vdash \varphi_{n}$ then we have $R \Vdash \varphi$

This is proved by induction on the proof of $\varphi_{1}, \ldots, \varphi_{n} \vdash \varphi$

Similar to the proof of soundness for Kripke/Beth models

Hence if we have $\vdash 1=0$ we have $R \Vdash 1=0$ for all finitely presented $k$-algebra $R$ 


\section{Soundness Theorem}

Lemma: If $R \rightarrow S$ and we have a covering $f_{i}: R \rightarrow R_{i}$ then we can find a corresponding covering $g_{i}: S \rightarrow S_{i}$ with commuting maps $h_{i}: R_{i} \rightarrow S_{i}$

Lemma: If $R \Vdash \varphi\left(a_{1}, \ldots, a_{n}\right)$ and $f: R \rightarrow S$ then $S \Vdash \varphi\left(f\left(a_{1}\right), \ldots, f\left(a_{n}\right)\right)$ 


\section{Site model}

Lemma: $R \Vdash a=0$ iff $a$ is nilpotent

Indeed, if $a$ is nilpotent in $R[X] /\langle p\rangle$ it is nilpotent in $R$ and if $a$ is nilpotent in $R\left[b^{-1}\right]$ and in $R /\langle b\rangle$ then it is nilpotent in $R$ 


\section{Site model}

We can see this forcing relation as defining one model, similar to Beth/Kripke model

This model (the "generic" model, similar to the initial model for equational theories) can be described in a weak metatheory (no axiom of choice)

This gives an effective consistency proof for the theory of algebraically closed fields

Indeed $R \Vdash 1=0$ iff $1=0$ in $R$

This builds a generic model, where the truth-values are non standard 


\section{Completeness Theorem}

We say that a formula $\varphi$ is positive iff it does not contain $\forall, \rightarrow$

$$
\varphi::=\perp|t=u| \varphi \wedge \varphi|\varphi \vee \varphi| \exists x . \varphi
$$

For a positive formula, a proof of $R \Vdash \varphi$ has a simple tree structure building a covering of $R$

We can see this as a cut-free proof of $\varphi$ 


\section{Completeness Theorem}

Two approaches for completeness

(1) Henkin-Lindenbaum

(2) Löwenheim-Skolem-Herbrand-Gödel, gives completeness of cut-free proofs 


\section{Completeness Theorem}

For positive formulae, to be true in a site model means to have a cut-free proof (well-founded tree)

Indeed, a proof theory with exactly this notion of proof tree is described in the paper

M. Coste, H. Lombardi and M.F. Roy, Dynamical method in algebra, Ann. Pure Appl. Logic 111 (2001), 203-256

The semantics is sound w.r.t. intuitionistic derivation, and the proof of soundness is similar to a proof of admissibility of the cut rule 


\section{Refinement of the model}

If we are at the node $R=k[x] /\left\langle x^{2}-3 x+2\right\rangle$ and we want to force $a=0 \vee \operatorname{Vinv}(a)$ for $a=x-3$

We can directly see that $a$ is invertible in $R$ by computing the GCD of $x^{2}-3 x+2$ and $x-3$

$$
x^{2}-3 x+2=x(x-3)+2
$$

so that the inverse of $a$ is $-x / 2$ 


\section{Refinement of the model}

Similarly for $a=x-1$ we find

$$
x^{2}-3 x+2=(x-1)(x-2)
$$

so that one branch is $R \rightarrow k[x] /\langle x-1\rangle$ where $a=x-1$ is 0 and the other branch is $R \rightarrow k[x] /\langle x-2\rangle$ where $a=x-1$ is invertible (and is equal to 1 )

$$
R\left[a^{-1}\right]=k[x] /\langle x-2\rangle \quad R /\langle a\rangle=k[x] /\langle x-1\rangle
$$




\section{Refinement of the model}

Finally in characteristic 0 (or over a perfect field) we can assume that we restrict the addition of roots to separable polynomials, by GCD computations

In this way, the nodes are all given by a finite number of indeterminates $x_{1}, \ldots, x_{n}$ and polynomial constraints

$$
p_{1}\left(x_{1}\right)=0, p_{2}\left(x_{1}, x_{2}\right)=0, \ldots, p_{n}\left(x_{1}, \ldots, x_{n}\right)=0
$$

and the algebra $R=k\left[x_{1}, \ldots, x_{n}\right] /\left\langle p_{1}, \ldots, p_{n}\right\rangle$ is $\mathrm{vN}$ regular 
Refinement of the model

The two covering relations are

$-R \rightarrow R_{0}=R /\langle e\rangle$ and $R \rightarrow R_{1}=R /\langle 1-e\rangle$, so that $R=R_{0} \times R_{1}$

$-R \rightarrow R[X] /\langle p\rangle$ where $p$ is separable 


\section{Refinement of the model}

For instance if $R=k[x, y] /\left\langle x^{2}-2, y^{2}-2\right\rangle$ and we want to force

$$
a=0 \vee \operatorname{inv}(a)
$$

for $a=y-x$ we get the covering

$$
R_{0}=k[x, y] /\left\langle x^{2}-2, y-x\right\rangle \quad R_{1}=k[x, y] /\left\langle x^{2}-2, y+x\right\rangle
$$




\section{Refinement of the model}

This gives a computational model of the algebraic closure of a field, for which we don't use a factorisation algorithm for polynomials over $k$, only GCD computation

This might be interesting even if we have a factorization algorithm for polynomials over $k$

One can think of each such finitely presented $k$-algebra as a finite approximation of the (ideal) algebraic closure of $k$ 


\section{Dynamical evaluation}

We get in this way what is known as dynamical evaluation in computer algebra (D. Duval; one application: computation of branches of an algebraic curves)

The notion of site model gives a theoretical model of dynamical evaluation

The same technique can be used for several other first-order theories

M. Coste, H. Lombardi and M.F. Roy, Dynamical method in algebra, Ann. Pure Appl. Logic 111 (2001), 203-256 


\section{Site model}

This is reminiscent of the description of Kronecker's work by $\mathrm{H}$. Edwards

The necessity of using an algebraically closed ground field introduced -and has perpetuated for 110 years- a fundamentally transcendental construction at the foundation of the theory of algebraic curves. Kronecker's approach, which calls for adjoining new constants algebraically as they are needed, is much more consonant with the nature of the subject

H. Edwards Mathematical Ideas, Ideals, and Ideology, Math. Intelligencer 14 (1992), no. 2, 6-19.

Cf. T. Mora Solving Polynomial Equation Systems I, The Kronecker-Duval Philosophy 


\section{Other theories}

Theory of local rings

$$
\operatorname{inv}(x) \vee \operatorname{inv}(1-x)
$$

where $\operatorname{inv}(u)$ means $\exists y .1=y u$

The elementary covering are now $R \rightarrow R\left[x^{-1}\right]$ and $R \rightarrow R\left[(1-x)^{-1}\right]$

Lemma: We have $R \Vdash i n v(x)$ iff $x$ is invertible in $R$

Lemma: We have $R \Vdash J(x)$ iff $x$ is nilpotent in $R$

Corollary: We don't have $\vdash \operatorname{inv}(x) \vee J(x)$ in the (intuitionistic) theory of local rings 


\section{Other theories}

It would be interesting to express similarly the theory of differential algebraic closure 


\section{Other theories}

"When Galois discussed the roots of an equation, he was thinking in term of complex numbers, and it was a long time after him until algebraist considered fields other than subfields of $\mathbb{C} \ldots$ But at the end of the century, when the concern was to construct a theory analogous to that of Galois, but for differential equations, they got stuck on the following problem: In what domain do we need to be in order to have enough solutions to differential equations? It was an important contribution of model theory to algebra to answer this question with the notion of differentially closed field which is to differential equations what the notion of algebraically closed field is to algebraic equations, a domain where differential equations have as many solutions as we can reasonable hope for. There is no natural example of a differentially closed field."

Constructively the problem appears already for the algebraic closure of a field 


\section{References}

J. Avigad. Forcing in proof theory. Bulletin of Symbolic Logic, 2001.

A. Boileau and A. Joyal. La logique des topos, Journal of Symbolic Logic 46, (1981), 6-16

M. Coste, H. Lombardi and M.F. Roy, Dynamical method in algebra, Ann. Pure Appl. Logic 111 (2001), 203-256

P. Johnstone Sketches of an elephant: a topos theory compendium, Vol. 2 


\section{Splitting field}

We have seen that there are problems to build the splitting field of $X^{2}+1$ over a field $k$

We can always build the $k$-algebra $R=k[X] /\left\langle X^{2}+1\right\rangle$

This splitting field exists in the topological model over the Zariski spectrum of $R$

This is a Boolean lattice, and the formula $x=0 \vee \exists y .1=x y$ is valid in this topological model 